\title{
Avaliação de grandezas elétricas na geração fotovoltaica para melhoria da eficiência por sistema de arrefecimento convectivo
}

\section{Mayara Cordeiro França, Luiz Antônio Pimentel Cavalcanti}

Instituto Federal de Educação, Ciência e Tecnologia da Bahia. Departamento de Ensino. Coordenação de Engenharia Elétrica. Av. Marcondes Ferraz, 200. General Dutra. Paulo Afonso-BA, Brasil (CEP 48607-000). E-mail: luiz.cavalcanti@ifba.edu.br.

Resumo. Fontes alternativas de geração de energia elétrica tem sido foco nos últimos anos visando a suprir o consumo energético mundial por vias sustentáveis para diminuir a dependência por combustíveis fósseis. Uma alternativa parte do efeito fotovoltaico, que converte diretamente a energia luminosa do sol (abundante e gratuita) em energia elétrica de forma silenciosa e estática. Este artigo avalia empiricamente os valores de tensão de curto circuito gerada pela placa para diferentes condições de temperatura, controlado através do arrefecimento convectivo da superfície de uma placa fotovoltaica. Tendo em vista que a Geração Distribuída tem se tornado evidente no cenário brasileiro e que a eficiência das placas depende não somente da sua construção química, mas também de fatores externos como a irradiância, a temperatura, o acúmulo de sujeira, sombreamento e inclinação da placa. 0 fator que mais afeta o Ponto de Potência Máximo $\left(\mathrm{P}_{\mathrm{MP}}\right)$ é a temperatura que para a placa utilizada consiste na variação de $2,55 \% /{ }^{\circ} \mathrm{C}$. Isto implica na necessidade de maximizar a eficiência da geração de energia elétrica por placas fotovoltaicas a partir do controle da temperatura. Assim, o sistema de arrefecimento convectivo por película d'água que reutiliza a água para arrefecer uma placa fotovoltaico se mostra como uma ótima solução para elevar a tensão de saída da placa em 1,02 V, consequentemente elevando $\mathrm{P}_{\mathrm{MP}}$.

Palavras-chave: Fontes alternativas; Eficiência de placa fotovoltaica; Sistema de arrefecimento.

Abstract. Evaluation of electric panels in the photovoltaic generation to improve efficiency by convective cooling system. Alternative sources of electricity generation have been focus in recent years aiming to supply the world's energy consumption by sustainable routes to reduce dependence on fossil fuels. An alternative part of the photovoltaic effect, which directly converts the luminous energy of the sun (abundant and free) into electrical energy in a silent and static way. This paper empirically evaluates the short-circuit voltage values generated by the plate for different temperature conditions, controlled by the convective
Recebido: $16 / 04 / 2018$

Aceito:

$27 / 04 / 2018$

Publicado:

$30 / 04 / 2018$

Acesso aberto

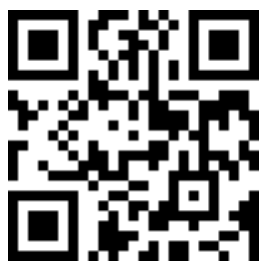

ORCID

(D) 0000-0003-2064-6768

Mayara Cordeiro

França

(1) 0000-0003-4932-9387

Luiz Antônio Pimentel

Cavalcanti 
cooling of the surface of a photovoltaic plate. Considering that Distributed Generation has become evident in the Brazilian scenario and that the efficiency of the plates depends not only on their chemical construction, but also on external factors such as irradiance, temperature, dirt accumulation, shading and slope of the plate. The factor that most affects the Maximum Power Point (PMP) is the temperature that for the plate used consists of the variation of $2.55 \% /{ }^{\circ} \mathrm{C}$. This implies the need to maximize the efficiency of electric power generation by photovoltaic panels from the temperature control. Therefore, the water film convective cooling system which reuses water to cool a photovoltaic plate is shown as an optimal solution to raise the plate output voltage by $1.02 \mathrm{~V}$, thereby raising PMP.

Keywords: Alternative sources; Photovoltaic system efficiency; Cooling system.

\section{Introdução}

Uma maior participação das fontes de energia renováveis na matriz energética tem sido incentivada em nível mundial, e uma série de políticas públicas vêm sendo adotadas por diferentes países a fim de se buscar maior segurança energética. Neste contexto, a energia solar fotovoltaica desempenha, potencialmente, um papel importante na evolução da participação de fontes alternativas na matriz energética mundial, visto sua abundância e ampla disponibilidade na superfície terrestre (Nakabayashi, 2014).

Através do efeito fotovoltaico, células solares convertem diretamente a energia do sol em energia elétrica de forma estática, silenciosa, não-poluente e renovável (Rüther, 2004). Segundo a Agência Nacional de Energia Elétricas (ANEEL), os maiores índices de radiação são observados na região Nordeste com destaque para o Vale do São Francisco. Este destaque pode ser melhor aproveitado através do controle da temperatura das placas fotovoltaicas.

Segundo Simioni (2017), os principais resultados obtidos mostram que o impacto da temperatura de operação das células fotovoltaicas é expressivo na identificação das regiões com maiores potenciais solares reais e que a eficiência elétrica pode ser reduzida em até $15 \%$. Matematicamente, Silva (2016) mostrou que derivando a fórmula do ponto máximo de potência é possível verificar a variação de potência dos módulos fotovoltaicos em relação a um gradiente de temperatura que resulta em $-0,46 \%$ por grau Celsius.

0 presente artigo tem como objetivo avaliar a eficiência de uma placa fotovoltaica a partir dos dados obtidos no projeto de um sistema de arrefecimento em função do controle da temperatura superficial da placa, partindo de um sistema sustentável que utilizou materiais reutilizados.

\section{Fundamentação teórica}

O desempenho real dos módulos fotovoltaicos é determinado pelas comdições ambientais, as quais podem causar efeitos que se traduzem em perda de eficiência. Dentre os principais fatores críticos para o desempenho dos módulos destacam-se: a temperatura de operação, irradiação, espectro solar, acúmulo de sujeira e sombreamento. Estes fatores são capazes de reduzir a eficiência de conversão em até $15 \%$ e podem levar a degradação das células e módulos (Simioni, 2017). 


\section{influência}

Parâmetros com maior

Existem dois parâmetros externos que mais afetam as características elétricas dos painéis fotovoltaicos: a irradiância solar e a temperatura, que é uma característica intrínseca à região de instalação das placas fotovoltaicas. 0 aumento da irradiância incidente e/ou da temperatura ambiente produz um aumento da temperatura da célula, e consequentemente tende a reduzir a sua eficiência (Pinho e Galdino, 2014). Como pode ser observado na Figura 1.

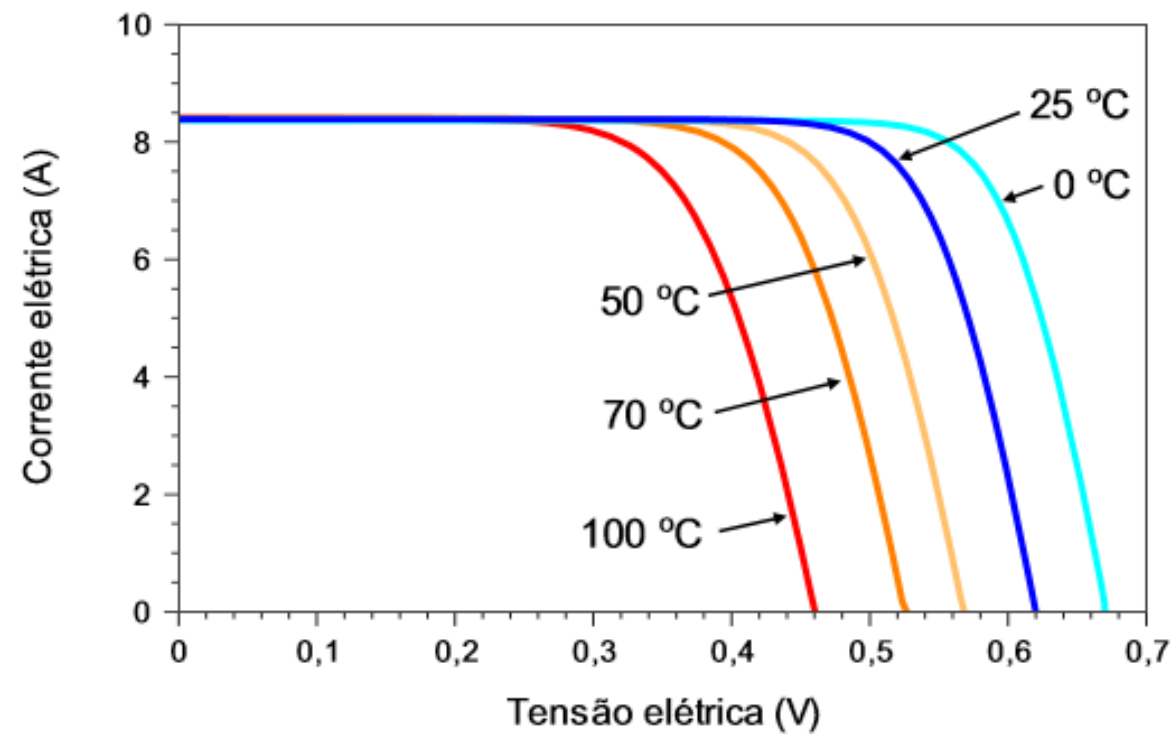

Figura 1. Relação entre a tensão e a corrente em função da temperatura nas placas. Fonte: Pinho e Galdino (2014).

Um modo de se analisar alguns dos fatores de perdas da instalação é observando a curva característica I-V do sistema, apresentado na Figura 2. Esta curva mostra os valores da corrente de saída de um conversor fotovoltaico, em função da sua tensão de saída, em condições preestabelecidas de temperatura e irradiância total. 0 Ponto de Potência Máxima $\left(\mathrm{P}_{\mathrm{MP}}\right)$ ou também denominado como MPP (Maximum Power Point) é o ponto da curva na qual o produto da corrente pela tensão é máximo (Urbanetz Jr., 2014; Rank, 2016). 


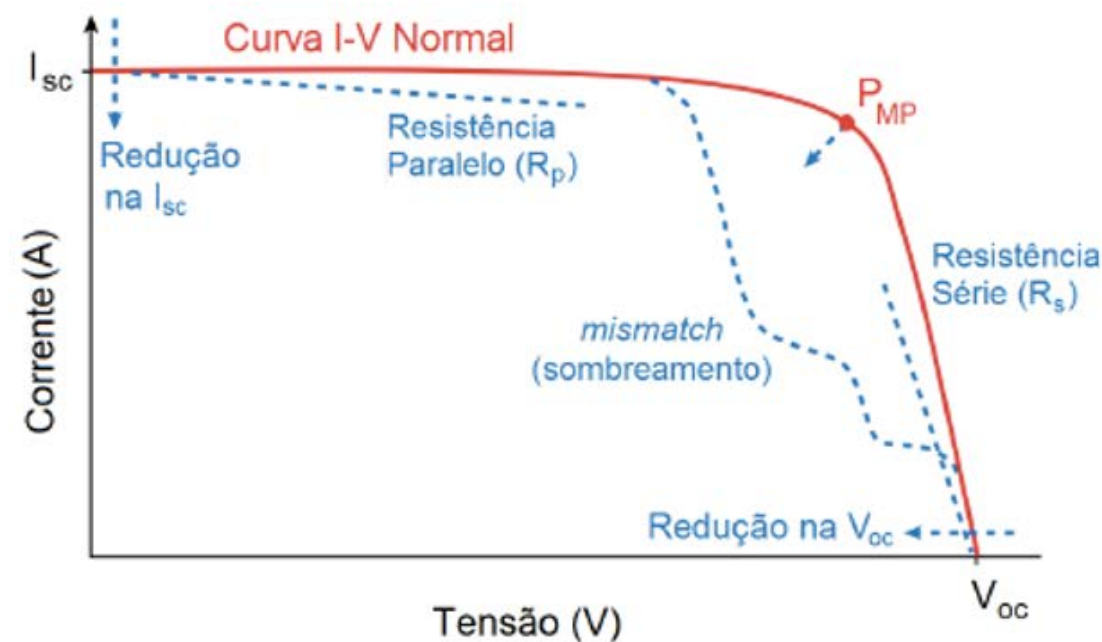

Figura 2. Anomalias da curva I-V de um painel fotovoltaico. Fonte: Pinho e Galdino (2014). 
A corrente gerada pela placa fotovoltaica apresenta poucas variações com a alteração da temperatura da célula, porém, com o aumento da temperatura da mesma, a tensão de circuito aberto do módulo fotovoltaico apresenta uma diminuição em seus valores muito mais significativa, em consequência do aumento da temperatura, além de ocorrer um deslocamento para baixo do ponto de máxima potência, este também é deslocado significativamente à esquerda, conforme observado na Figura 3. 0 aumento da temperatura faz com que a banda de energia do material semicondutor diminua, resultando em um acréscimo da fotocorrente gerada, de aproximadamente $0,1 \%$. Entretanto, a tensão de circuito aberto, decresce a uma taxa de $0,3 \% /{ }^{\circ} \mathrm{C}$ (Seguel, 2009).

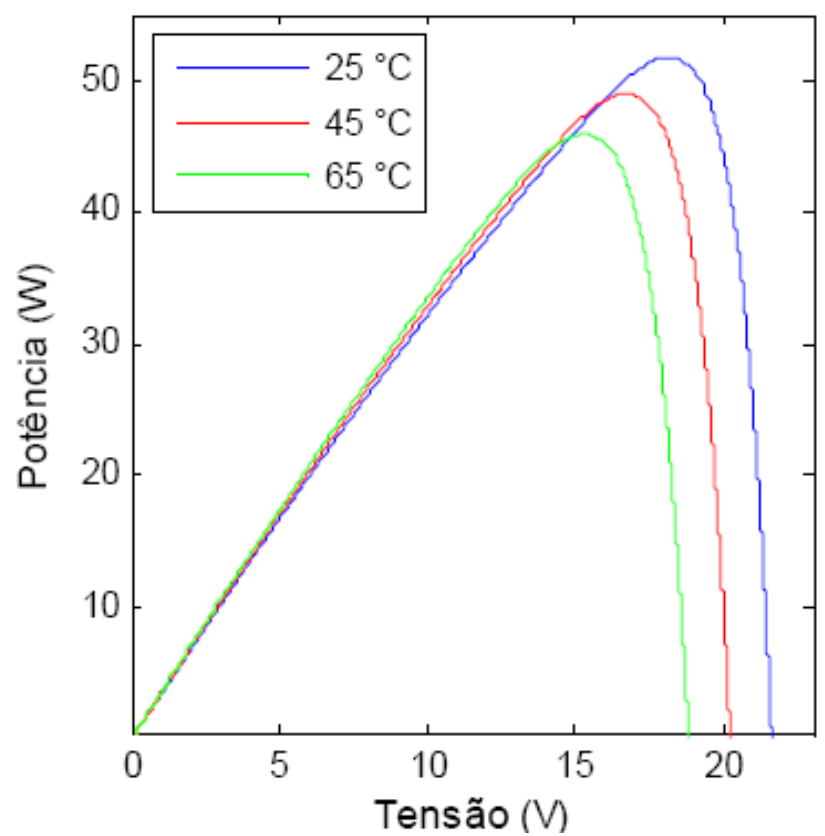

Figura 3. Curva P-V para diferentes temperaturas. Fonte: Seguel (2009).

\section{0 modelo elétrico equivalente}

A corrente em célula fotovoltaica pode ser considerada como a soma de corrente de uma junção $p n$ no escuro (diodo semicondutor) com a corrente gerada pelos fótons absorvidos da radiação solar (Pinho e Galdinho, 2014). Podendo ser descrita a partir da Equação de Schockley de diodo, Equação 1.

$$
I=I_{L}-I_{0}\left[\left(e^{\frac{q V}{n k T}}\right)-1\right]
$$

Onde:

$\mathrm{I}_{\mathrm{L}}$ - Corrente fotogerada $(\mathrm{A})$;

$\mathrm{I}_{0}$ - Corrente de Saturação reversa do diodo (A);

n - Fator de idealidade do diodo, número adimensional geralmente entre 1 e 2 , obtido por ajuste de dados experimentais medidos;

q - Carga do elétrons $\left(1,6 \times 10^{-19} \mathrm{C}\right)$;

$\mathrm{k}$ - Constante de Boltzman $\left(1,38 \times 10^{-23} \mathrm{j} / \mathrm{K}\right)$;

$\mathrm{T}$ - Temperatura absoluta. 
Podemos observar a partir da fotovoltaica se comportará como um Equação 1 que se $\mathrm{I}_{\mathrm{L}}$ for zero a célula diodo.

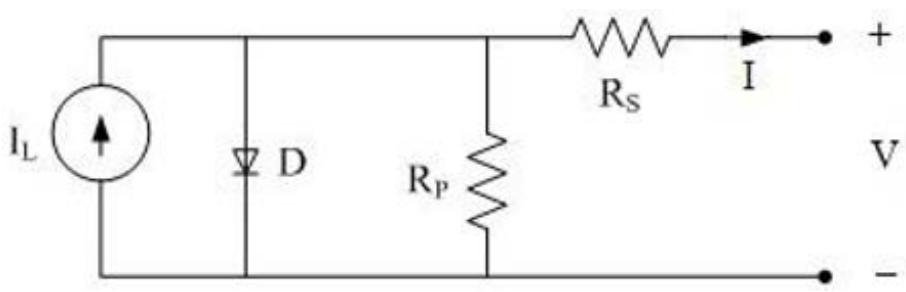

Figura 4. Circuito equivalente com um diodo para célula fotovoltaica. Fonte: Pinho e Galdinho (2014).

Os componentes descritos na Figura 4 que descrevem os seguintes fenômenos, segundo Silva (2016):

Rs - Descreve as perdas ôhmicas do material semicondutor, nas conexões em geral;

$\mathrm{Rp}$ - Descreve as perdas que surgem principalmente através das perturbações elétricas causadas pelas impurezas e defeitos da estrutura cristalina;

$\mathrm{I}_{\mathrm{L}}$ - representa a corrente a uma determina isolação;
D - a junção P-N;

V - A tensão de saída nos terminais da célula.

Como observado no circuito equivalente existe uma resistência em série - $R_{S}$ devido a junção metalsemicondutor, malhas metálicas, regiões dopadas, etc e uma resistência em paralelo $R_{P}$ proveniente de ponto de curto-circuito na junção pn, Equação 2.

$$
I=I_{L}-I_{0}\left[e^{\frac{q\left(V+I R_{S}\right)}{n k T}}-1\right]-\frac{V-I R_{S}}{R_{P}}
$$

Ao termo exponencial acrescentou a queda de tensão no resistor em série e a fórmula inicial foi diminuído a corrente relativa a resistência em paralelo. Para o modelo elétrico, estes termos podem ser considerados as perdas elétricas da placa fotovoltaica.

Uma única célula fotovoltaica, isoladamente, tem capacidade reduzida de produção de energia elétrica, tipicamente entre 1 e $2 \mathrm{~W}$, correspondente a uma tensão de $0,5 \mathrm{~V}$ e uma corrente entre 2 e 4 A. Portanto, para atingir determinados níveis de tensão e corrente, faz-se necessária a associação de várias células, através de ligações série e paralelo, formando os painéis fotovoltaicos (Seguel, 2009).

A Equação 2, segundo Gow e Manning (1999) e Seguel (2009), irá sofrer as modificações apresentadas na Equação 3, a depender da quantidade de células conectadas em paralelo e em série.

$$
I=n_{P}\left\{I_{L}-I_{0}\left[e^{\frac{q\left(\frac{V}{n_{S}}+\frac{I R_{S}}{n_{P}}\right)}{n k T}}-1\right]-\frac{\frac{V}{n_{S}}-\frac{I R_{S}}{n_{P}}}{R_{P}}\right\}
$$


Onde:

$\mathrm{n}_{\mathrm{P}}$ - Número de células conectadas em paralelo;

$\mathrm{n}_{\mathrm{s}}$ - Número de células conectadas em série.

\section{Parâmetros elétricos}

A partida a Figura 4 que apresenta a curva I-V com os principais parâmetros elétricos que caracterizam os módulos fotovoltaicos: tensão de circuito aberto, corrente de curto-circuito, fator de forma e eficiência (Pinho e Galdinho, 2014).

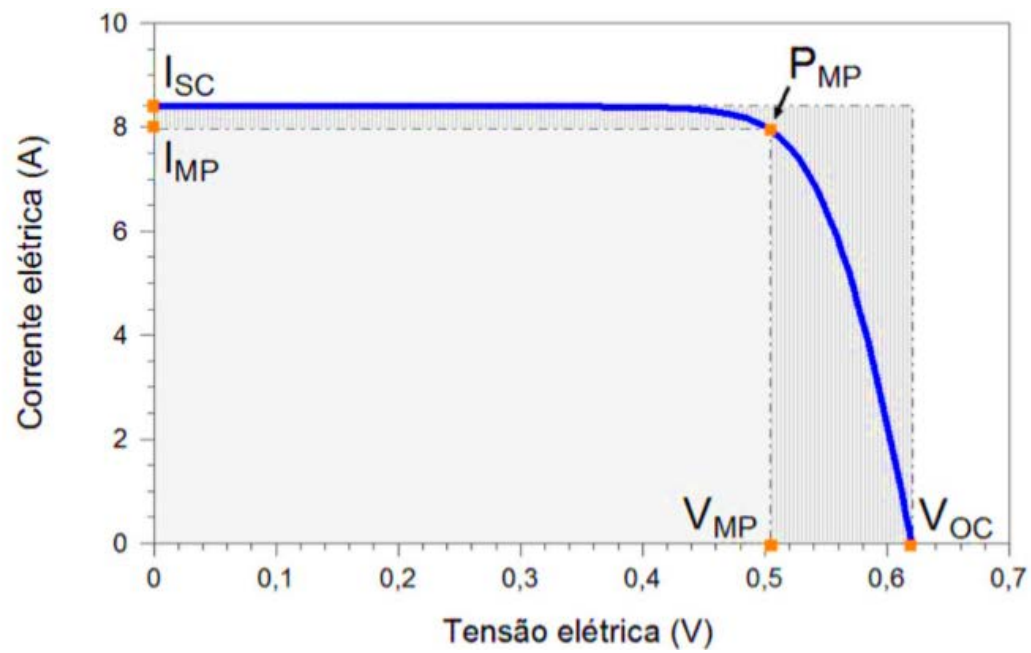

Figura 4. Principais parâmetros elétricos em destaque. Fonte: Pinho e Galdino (2014).

- Tensão de circuito aberto $\left(\mathrm{V}_{\mathrm{oc}}\right)$ : é a tensão entre os terminais de uma célula fotovoltaica quando não há corrente elétrica circulando e é a máxima tensão que uma célula fotovoltaica pode produzir.

- Corrente de curto-circuito $\left(\mathrm{I}_{\mathrm{sc}}\right)$ : é a máxima corrente que se pode obter $\mathrm{e}$

$$
F F=\frac{V_{M P} I_{M P}}{V_{\sigma \sigma} I_{s G}}
$$

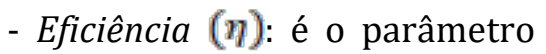
que define quão efetivo é o processo de conversão de energia solar em energia elétrica. Representa a relação entre a

$$
\eta=\frac{I_{s c} V_{o c} F F}{A G} \times 100 \%
$$

Onde:

é medida na célula fotovoltaica quando a tensão elétrica em seus terminais é igual a zero.

- Fator de forma (FF): é a razão entre a máxima potência da célula e o produto da corrente de curto circuito com a tensão de circuito aberto, Equação 4.

potência elétrica produzida pela célula fotovoltaica e a potência da energia solar incidente e pode ser definida como Equação 5:

$A\left(\mathrm{~m}^{2}\right)$ é a área da célula;

$\mathrm{G}\left(\mathrm{W} / \mathrm{m}^{2}\right)$ é a irradiância solar incidente. 


\section{Materiais e métodos}

O sistema de arrefecimento foi confeccionado com uma bomba d'água do tipo Submersible Pump, modelo SP-500 de marca JAD com potência de $6 \mathrm{~W}$ que consegue elevar a água até no máximo $60 \mathrm{~cm}$ de altura. Utilizou-se um recipiente retangular plástico de dimensões $9 \times 33 \times 54 \mathrm{~cm}$ para o reservatório d'água e uma mangueira de $150 \mathrm{~cm}$ para despejar a água sobre a superfície da placa (Figura 5).

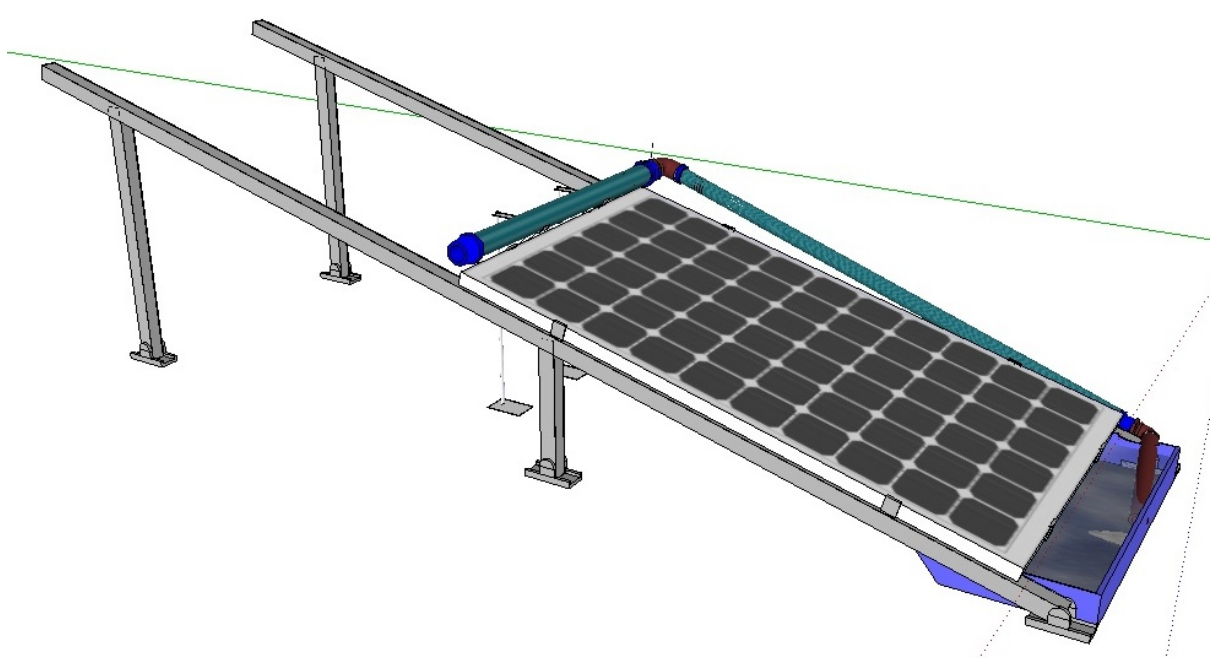

Figura 5. Sistema de resfriamento por película d'água protótipo montado no SketchUp Pro 2017.

O principal componente para funcionamento do sistema é a bomba d'água de aquário que tem como função direcionar a água que está no reservatório inferior para a borda superior da placa, onde a mangueira é fixada como mostra a Figura 6, e assim despejar pela mangueira com furos de 2 mm espaçados simetricamente a água despejada cria uma fina película d'água.

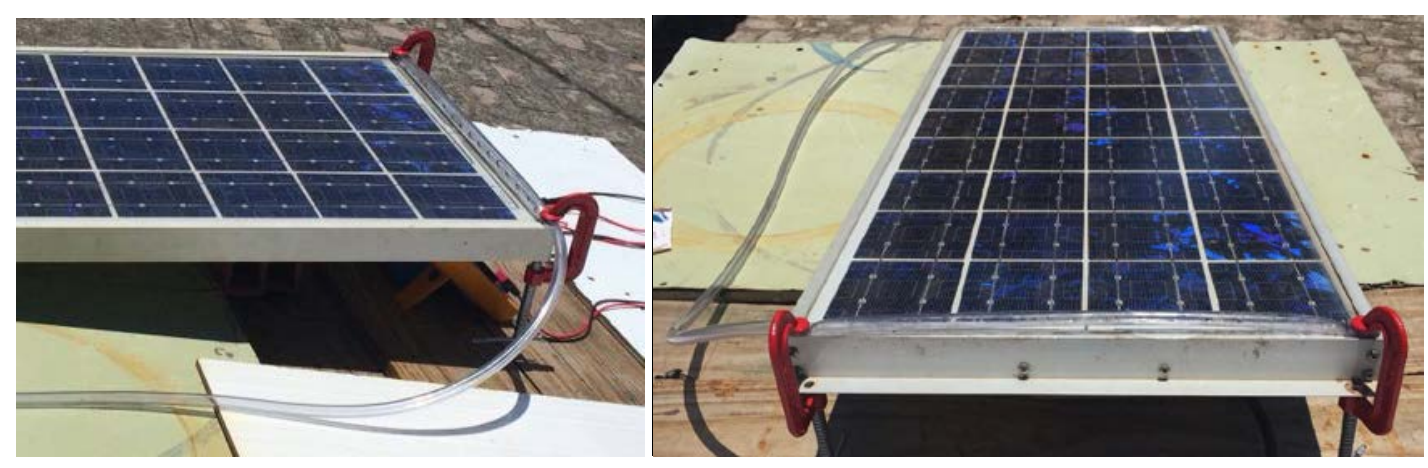

Figura 6. Sistema de fixação da mangueira com furos espaçados regularmente. 
Ao protótipo criado no SketchUp, Figura 5, acrescentou-se um protótipo de torre de arrefecimento para aumentar a troca de calor da água com por meio de um sistema reutilizado de latinha de alumínio, Figura 7, através do material metálico aumenta da troca térmica.

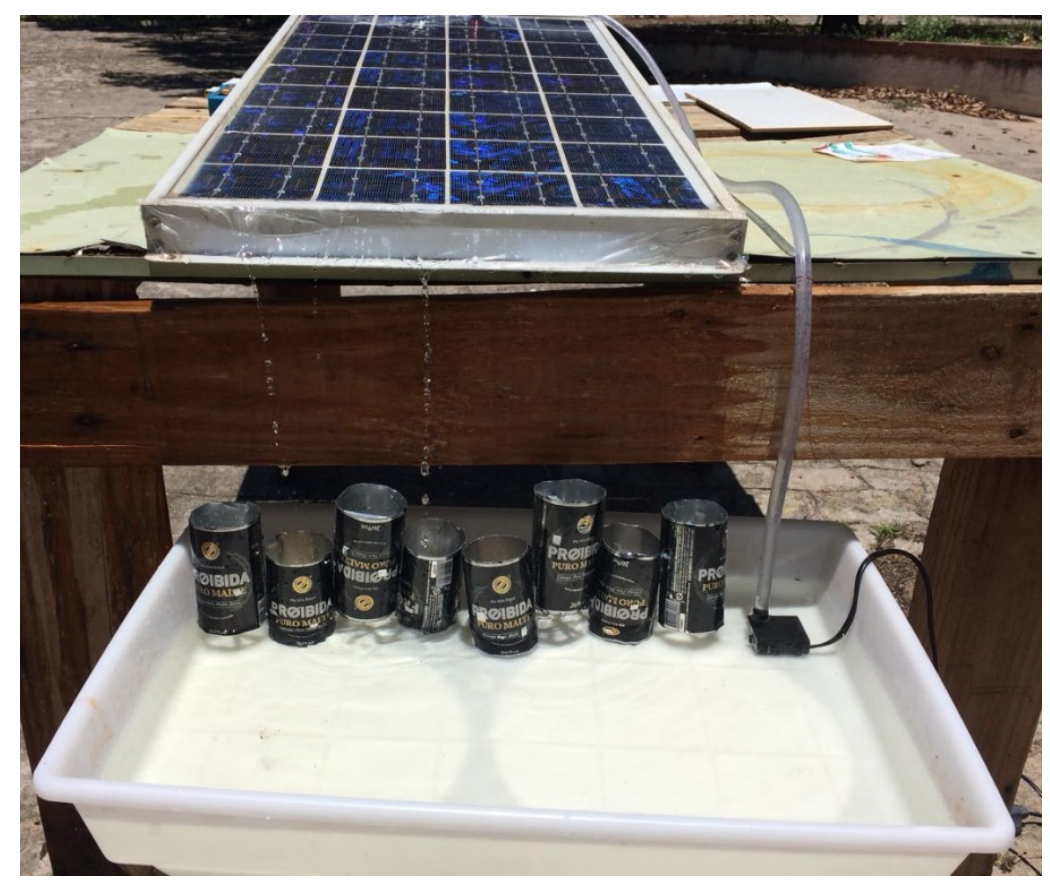

Figura 7. Sistema de resfriamento montado a partir de latas de alumínio reutilizadas.

A carga utilizada foi uma associação de dois (02) resistores em paralelo de $25 \Omega$ que resultou numa resistência equivalente de $13,3 \Omega$ mostrado na Figura 8. Com esta carga, aferiu-se a Tensão na carga e a corrente e se pode aproximar a potência ganha no sistema.

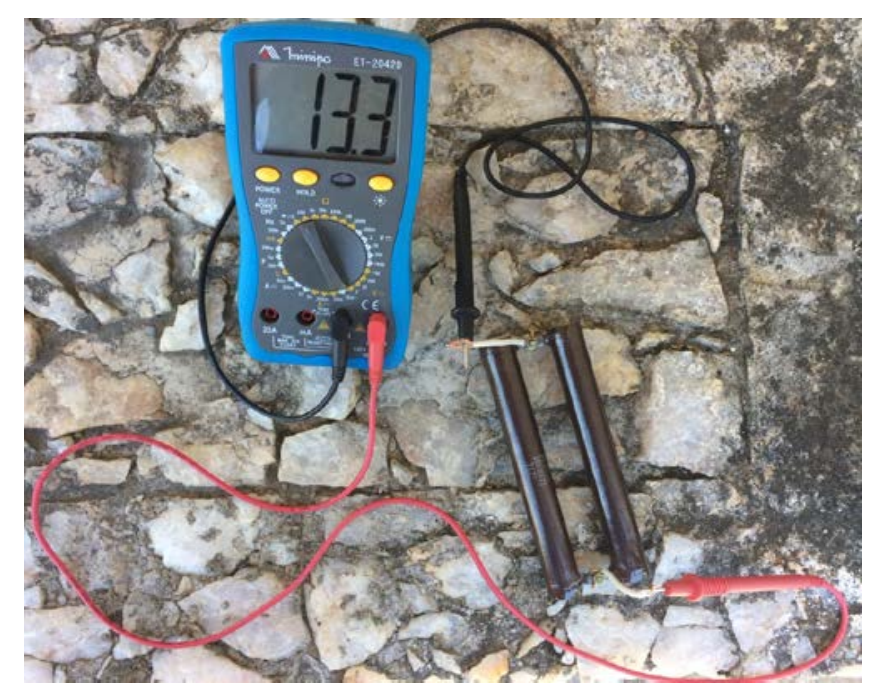

Figura 8. Associação em paralelo de dois resistores de $25 \Omega$. 
Para realizar a análise estatística do experimento utilizou-se o teste quiquadrado $\left(x^{2}\right)$, que segundo Conti (2009) é um teste de hipóteses que se destina a encontrar um valor da dispersão para duas variáveis nominais, avaliando a associação existente entre variáveis qualitativas. 0 princípio básico deste método é comparar proporções, isto é, as possíveis divergências entre as frequências observadas e esperadas para um certo evento. Evidentemente, pode-se dizer que dois grupos se comportam de forma semelhante se as diferenças entre as frequências observadas e as esperadas em cada categoria forem muito pequenas, próximas a zero. Este teste pode ser utilizado para:

- Verificar se a frequência com que um determinado acontecimento observado em uma amostra se desvia significativamente ou não da frequência com que ele é esperado.

- Comparar a distribuição de diversos acontecimentos em diferentes amostras, a fim de avaliar se as proporções observadas destes eventos mostram ou não diferenças significativas ou se as amostras diferem significativamente quanto às proporções desses acontecimentos.

\section{Medições das variáveis elétricas}

As medições foram realizadas no campus do Sal Torrado do IFBA no Município de Paulo Afonso, Estado da Bahia. Sua localização, observada na Figura 9, possui latitude de 9,38170334 ${ }^{\circ}$ $S$ e longitude de $38.22547495^{\circ} \mathrm{O}$ e elevação de $254 \mathrm{~m}$, segundo dados do Google Maps.

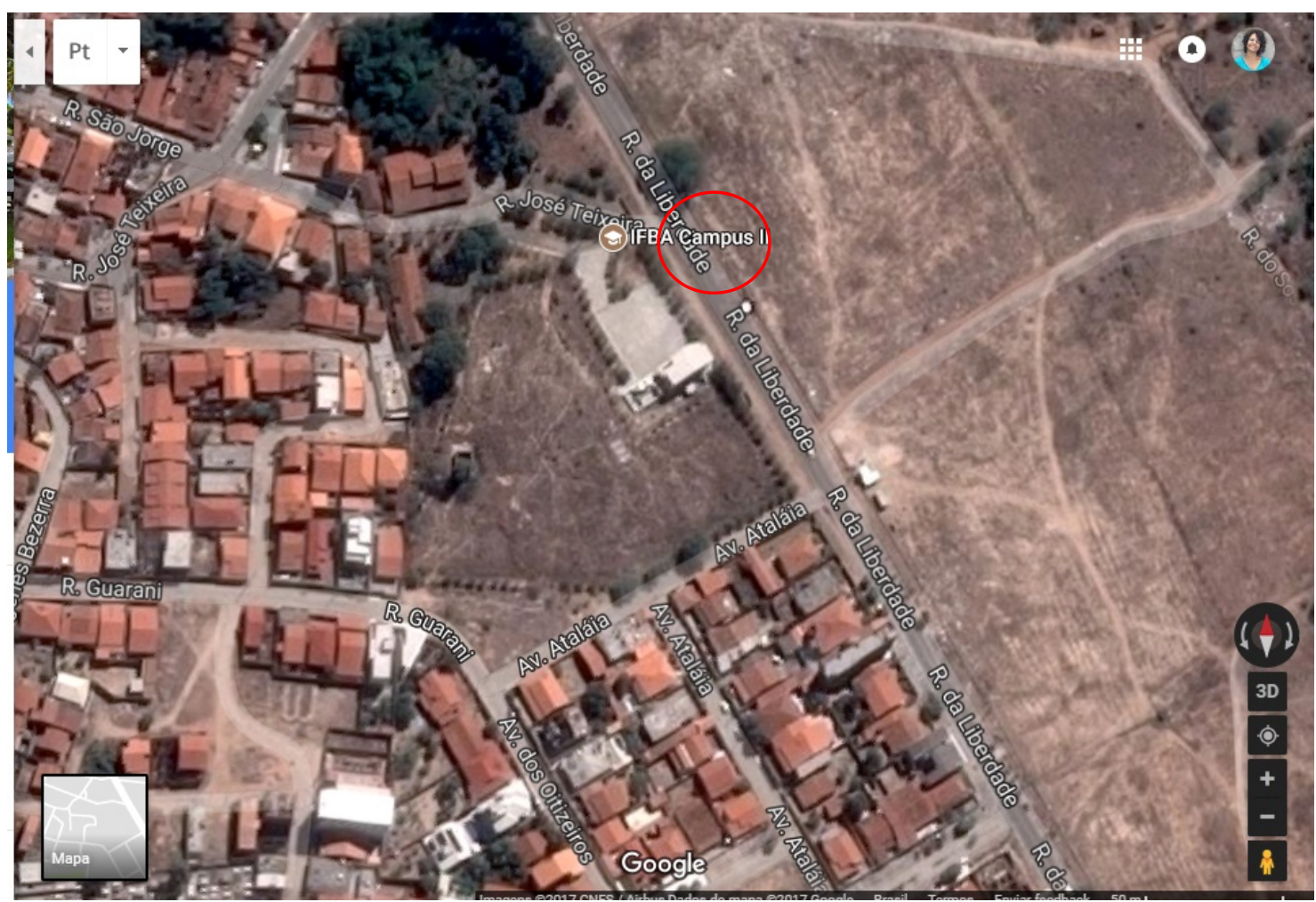

Figura 9. Localização da realização das medições. Fonte: Google Maps (2017). 
Os valores da temperatura e irradiância ao longo do ano para qualquer sítio no Brasil são fornecidos pelo Centro de Referência para as Energias Solar e Eólica Sérgio de Salvo Brito (CRESESB), do Centro de Pesquisas de Energia Elétrica (CEPEL), que disponibiliza o programa Sundata. A partir deste banco de dados do site
CRESESB com a entrada das informações de latitude e longitude, pode-se determinar a angulação adequada para posicionamento da placa. Observando a Tabela 1, considera-se qual a maior média anual de irradiância incidente no Município de Paulo Afonso, mostrada na Figura 10.

Tabela 1. Valores de irradiação média mensal para a localização de realização das medições.

\begin{tabular}{|c|c|c|c|c|c|c|c|c|}
\hline \multirow{2}{*}{ Ângulo } & \multirow{2}{*}{ Inclinação } & \multicolumn{7}{|c|}{ Irradiação solar diária média mensal (kW/m².dia) } \\
\hline & & Jan & $\mathrm{Fev}$ & Mar & Abr & Mai & Jun & Jul \\
\hline Plano horizontal & $0^{\circ} \mathrm{N}$ & 5,78 & 5,67 & 5,69 & 5,03 & 4,36 & 4,06 & 4,31 \\
\hline Ângulo igual a latitude & $9^{\circ} \mathrm{N}$ & 5,49 & 5,51 & 5,7 & 5,21 & 4,64 & 4,38 & 4,63 \\
\hline Maior média anual & $6^{\circ} \mathrm{N}$ & 5,6 & 5,57 & 5,71 & 5,16 & 4,56 & 4,28 & 4,53 \\
\hline Maior mínimo mensal & $25^{\circ} \mathrm{N}$ & 4,77 & 5,01 & 5,46 & 5,29 & 4,93 & 4,76 & 4,99 \\
\hline \multirow{2}{*}{ Ângulo } & \multirow{2}{*}{ Inclinação } & \multicolumn{7}{|c|}{ Irradiação solar diária média mensal (kW/m².dia) } \\
\hline & & Ago & Set & Out & Nov & Dez & Média & Delta \\
\hline Plano horizontal & $0^{\circ} \mathrm{N}$ & 5,25 & 5,44 & 6,36 & 6,5 & 6,17 & 5,39 & 2,44 \\
\hline Ângulo igual a latitude & $9^{\circ} \mathrm{N}$ & 5,52 & 5,52 & 6,23 & 6,19 & 5,81 & 5,4 & 1,85 \\
\hline Maior média anual & $6^{\circ} \mathrm{N}$ & 5,44 & 5,5 & 6,29 & 6,3 & 5,94 & 5,41 & 2,02 \\
\hline Maior mínimo mensal & $25^{\circ} \mathrm{N}$ & 5,75 & 5,41 & 5,73 & 5,39 & 4,96 & 5,2 & 0,99 \\
\hline
\end{tabular}

Fonte: CRESESB (2017).

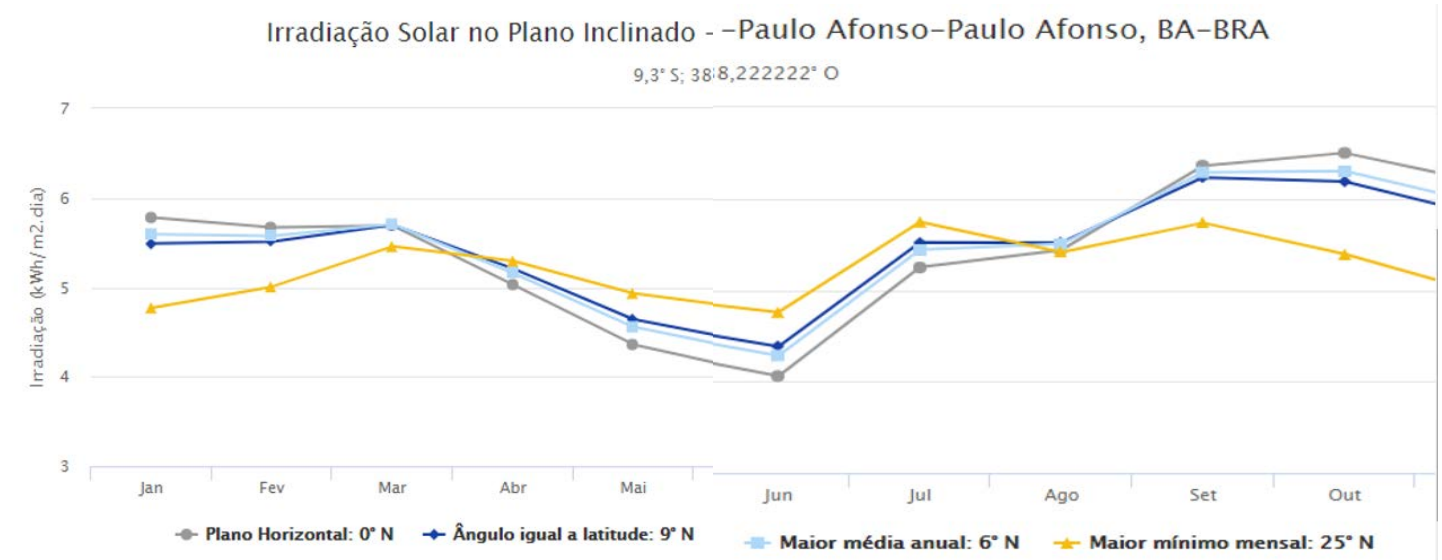

Figura 10. Irradiação solar por plano inclinado na cidade de Paulo Afonso. Fonte: CRESESB (2017).

A partir dos dados acima, optouse pela inclinação de $6^{\circ}$ a Norte posição que encontra maior média anual de irradiação. 0 sistema está montado sobre uma mesa e com um suporte superior para realizar a inclinação desejada.
A aferição das variáveis elétricas foi realizada com o uso de dois multímetros, um de marca Hikari, modelo HM-1100, para aferir os valores de tensão de circuito aberto $\left(\mathrm{V}_{\mathrm{oc}}\right)$ e outro de marca Minipa, modelo ET2042D, para 
aferir os valores de corrente de curtocircuito $\left(\mathrm{I}_{\mathrm{sc}}\right)$, tais variáveis elétricas sem carga, aferido nos terminais de saída da placa fotovoltaica. $\mathrm{O}$ mesmo foi realizado com a carga, obtendo os valores de corrente de carga e tensão de carga.

Com o termômetro digital infravermelho com mira a laser Minipa, modelo MT-350, aferiu-se a temperatura da placa, realizado em 3 pontos (início, meio e fim) e obtendo a média como resultado. Com os valores adquiridos foram construídas curvas em relação da tensão de curto circuito $\left(\mathrm{V}_{\mathrm{oc}}\right)$ medida versus a temperatura da placa.

\section{Resultados e discussão}

Utilizou-se a água para ser despejada sobre a placa tendo como função trocar calor com a superfície da mesma, a fim de diminuir a temperatura superficial e aumentar a Tensão de Circuito Aberto $\left(\mathrm{V}_{\mathrm{oc}}\right)$, variável elétrica que possui maior influência da temperatura.

Observando os valores da temperatura da placa ( $\mathrm{T}_{\text {placa }}$ ) variando a cada $10{ }^{\circ} \mathrm{C}$; considerou-se 5 temperaturas a partir do $60^{\circ} \mathrm{C}$ e comparando com os valores de $V_{\text {oc }}$ resultantes, mostrados na Tabela 2 , nota-se que a menor temperatura de funcionamento da placa, correspondente a $20^{\circ} \mathrm{C}$, equivale a um maior valor na $\mathrm{V}_{\text {oc. }}$ Comparando a temperatura da placa e a temperatura do chão podemos notar que a incidência da radiação solar permaneceu relativamente constante, tendo em vista que a temperatura do chão não sofreu grande variação durante os experimentos.

Tabela 2. Valores aferidos das grandezas elétricas e da temperatura.

\begin{tabular}{ccccccc}
$\mathbf{V}_{\mathbf{o c}}(\mathbf{V})$ & $\mathbf{I}_{\text {sc }}(\mathbf{A})$ & $\mathbf{V}_{\text {carga }}(\mathbf{V})$ & $\mathbf{I}_{\mathbf{c a r g a}}(\mathbf{A})$ & $\mathbf{T}_{\text {placa }}\left({ }^{\circ} \mathbf{C}\right)$ & $\mathbf{T}_{\text {chão }}$ & $\mathbf{T}_{\text {água }}$ \\
\hline 19,42 & 3,24 & 19,26 & 0,69 & 60 & 58 & 70 \\
\hline 19,58 & 3,19 & 19,14 & 0,71 & 50 & 58 & 33 \\
\hline 20,12 & 3,17 & 19,50 & 0,75 & 40 & 59 & 33 \\
\hline 20,30 & 3,16 & 19,62 & 0,76 & 30 & 52 & 29 \\
\hline 20,44 & 3,15 & 19,81 & 0,77 & 20 & 56 & 9 \\
\hline
\end{tabular}

Pode-se observar ainda que a temperatura da água despejada sobre a placa modificou proporcionalmente em relação a temperatura que se desejava atingir. Por este motivo que o sistema de arrefecimento conta com um protótipo que atue como uma torre de arrefecimento para induzir, também por convecção, a diminuição da temperatura da água; consequentemente da superfície da placa fotovoltaica. Este sistema é visto na Figura 11, utilizou-se o princípio dos radiadores para aumentar a superfície de contador com aletas internas para assim aumentar a troca térmica.

A Figura 12 exibe uma curva dos pontos experimentais de $V_{o c}$ em função da temperatura da placa. Observa-se, na Tabela 4, que o ajuste obtido por regressão linear apresentou coeficiente de correlação de $94,44 \%$ para um intervalo de confiança de $95 \%$. Os valores de tensão medidos e obtidos via modelo possui um erro padrão de 0,1226 para 5 observações realizadas.

0 Teste Qui-quadrado $\left(X^{2}\right)$ foi utilizado para comparar a dispersão entre a temperatura da placa e a tensão de circuito aberto, avaliando a associação existente entre as duas variáveis, por meio da comparação das proporções entre elas, ou seja, as possíveis divergências entre elas tendo em conta a frequência de ocorrência e observada se as diferenças entre as frequências observadas são muito pequenas, aproximando-se de zero. 


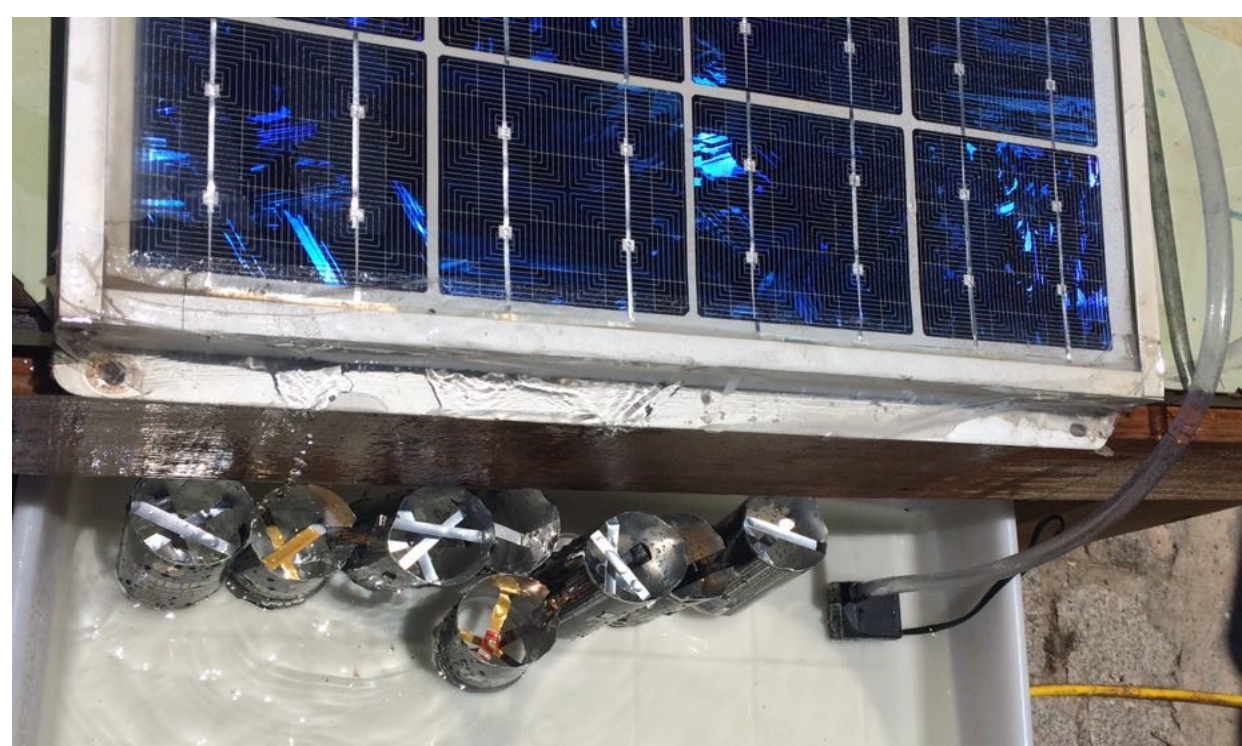

Figura 11. Visão superior das aletas internas do protótipo de resfriamento.

No teste de aderência do modelo, obteve na Tabela 3 os resultados, estes indicam que este modelo é indicado para representar os dados experimentais, uma vez que $\chi_{C}^{2}<\chi_{T}^{2}$; Estatisticamente os valores não são significativamente diferentes.

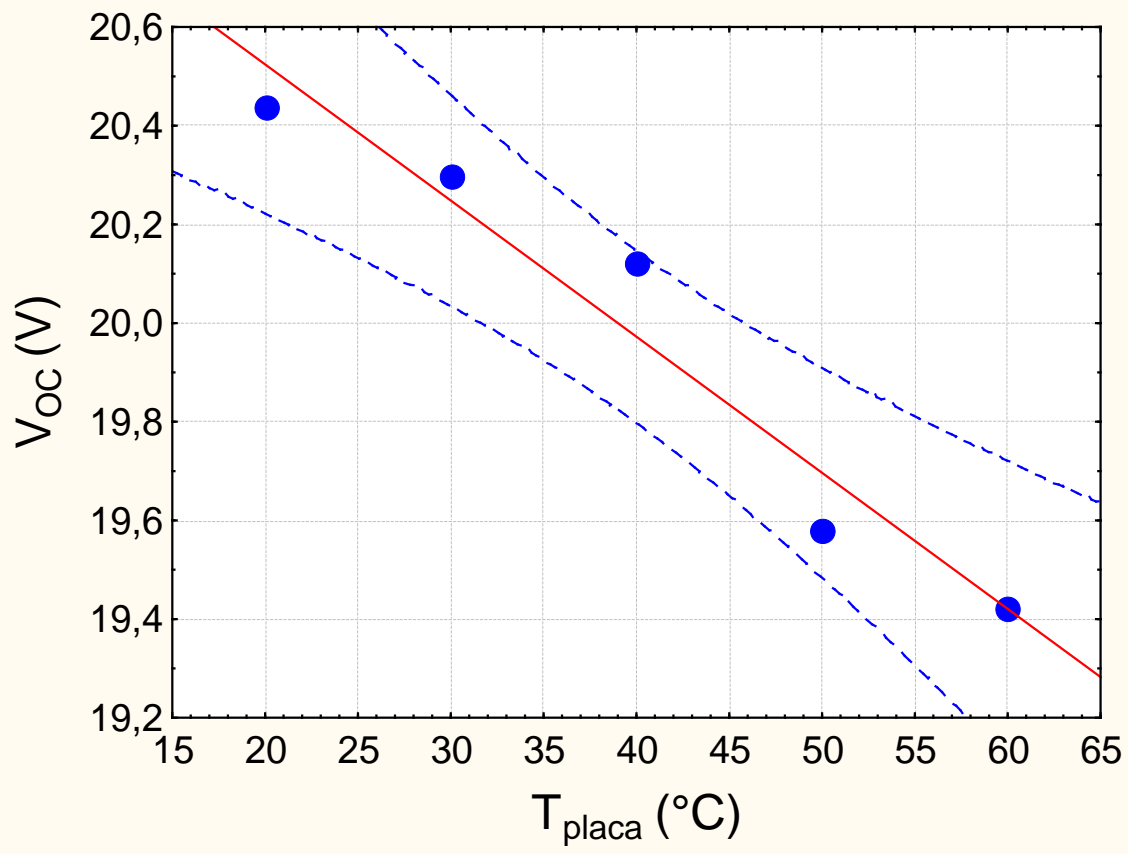

Figura 12. Variação da tensão $\left(\mathrm{V}_{\mathrm{oc}}\right)$ com a temperatura por regressão linear. 
Tabela 3. Resultado do teste de aderência Qui-quadrado.

\begin{tabular}{|l|c|}
\hline \multicolumn{2}{|c|}{ Teste de aderência do teste Qui-quadrado } \\
\hline $\mathrm{X}_{\text {cal }}$ & 0,0023 \\
\hline $\mathrm{X}_{\text {tab }}$ & 7,8147 \\
\hline
\end{tabular}

Inserindo os valores de temperatura e tensão no software Statistica do StatSoft ${ }^{\circledR}$ versão do estudante, obtemos um modelo mostrado na Equação 6. Sendo a variável ' $x$ ' a entrada para temperatura em grau Celsius e a saída da equação a tensão de circuito aberto em volts.

$$
V_{O C}(V)=21,076-0,0276^{*} x
$$

Analisando os resíduos, Figura 13 , observa-se que os valores de tensão oscilam próximo a zero em relação com a temperatura e que a discrepância não é superior a $0,16 \mathrm{~V}$. Isto sugere que o modelo possui boa aderência e que a relação entre as variáveis apresentadas mostra baixa divergência, inferindo que o modelo proposto representado nos dados experimentais obtidos possuem ótima aproximação.

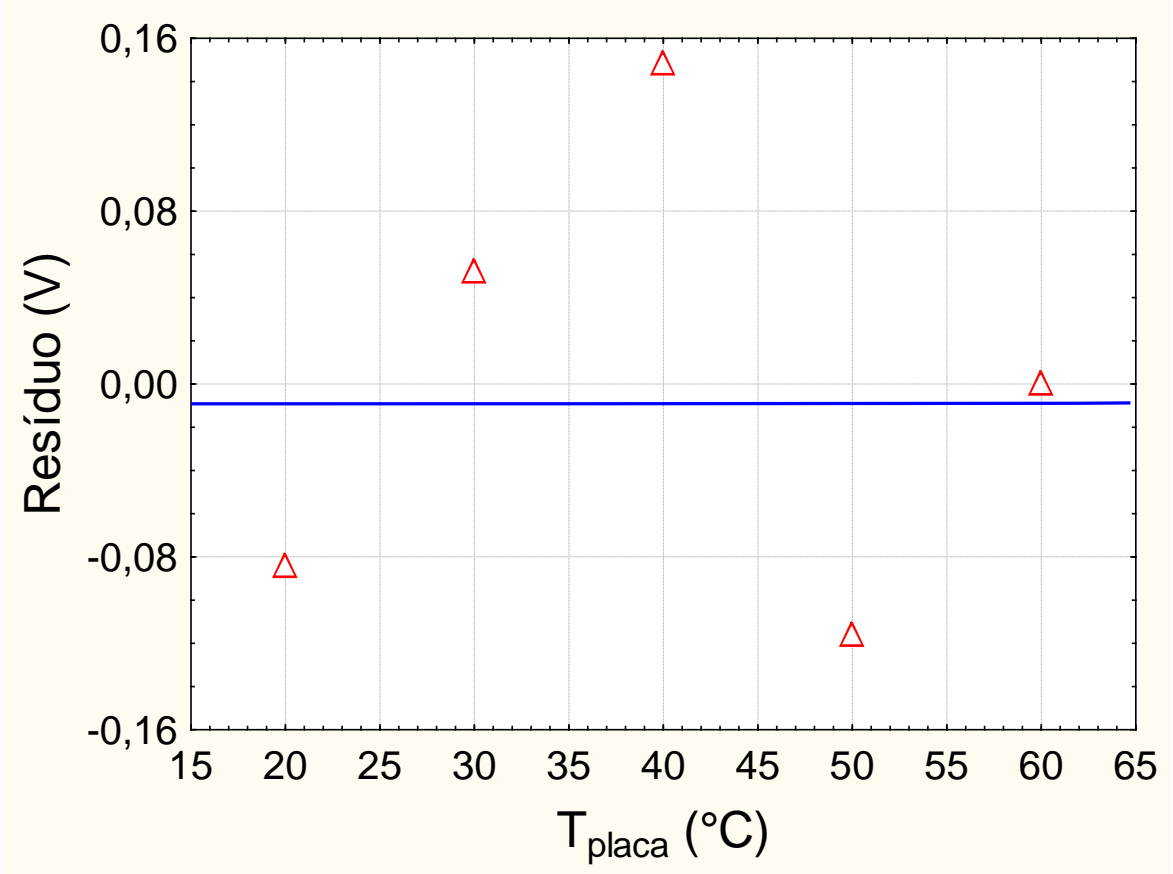

Figura 13. Resíduos resultantes do modelo.

\section{Conclusões}

Tendo em vista a influência da temperatura de operação na variável elétrica analisada, tensão de curto circuito (Voc), este trabalho buscou demonstrar experimentalmente que um sistema auxiliar de arrefecimento aplicado a uma placa fotovoltaica pode aumentar a tensão de saída da mesma utilizando um sistema de ciclo fechado para o líquido arrefecedor.

O sistema de arrefecimento se mostrou eficiente no que tange o aumento da tensão de curto circuito. Foi 
possível verificar o ganho de $1,02 \mathrm{~V}$ da tensão de saída pela diferença de temperatura de $40^{\circ} \mathrm{C}$. Como a geração por placas fotovoltaicas ainda possui baixa eficiência, os estudos devem avançar para tornar a implantação mais financeiramente viável.

A superfície terrestre possui um grande potencial para a geração fotovoltaica. Os investimentos nesta área se tornam cada dia maiores por ser uma energia limpa e sustentável. Buscar por meio de sistemas que melhorem a eficiência dos painéis fotovoltaicos é uma forma de contribuir a maior disseminação dessa fonte alternativa de geração de energia elétrica.

\section{Declaração de conflito de interesses}

Os autores declaram não haver conflito de interesses.

\section{Referências}

ANEEL - Agência Nacional de Energia Elétrica. Energia Solar 3. Disponível em: $<$ http://www2.aneel.gov.br/aplicacoes/atlas /pdf/03-energia_solar(3).pdf >. Acesso em: 25 abr. 2017.

Conti, F. Biometria qui-quadrado. Belém: Laboratório de Informática, ICB, UFPA, 2009. Disponível em: <http://www.ufpa.br/dicas/ biome/biopdf/bioqui.pdf $>$. Acesso em: 24 mar. 2018.

Gow, J. A.; Manning, C. D. Development of a photovoltaic array model for use in power electronics simulation studies. IEE Proceedings on Electric Power Applications, v. 146, n. 2, p. 193-200, 1999. https://doi.org/10.1049/ip-epa:19990116

Nakabayashi, R. Microgeração fotovoltaica no Brasil: condições atuais e perspectivas futuras. São Paulo: Universidade de São Paulo, 2014. (Dissertação de mestrado). Disponível em: <http://www.iee.usp.br/lsf/
sites/default/files/Dissertacao_Rennyo_vfina l.pdf>. Acesso em: 02 maio 2017.

Pinho, J. T.; Galdino, M. A. Manual de engenharia para sistemas fotovoltaicos. Rio de Janeiro: CEPEL/CRESESB, 2014. Disponível em: <http://www.cresesb.cepel. $\mathrm{br} /$ publicacoes/download/Manual_de_Engen haria_FV_2014.pdf>. Acesso em: 12 abr. 2017.

Rank, N. I.; Araújo, A. J. N; Bueno, T. B. A. Análises dos fatores de perdas nos sistemas fotovoltaicos conectados á rede elétrica em Curitiba. 2016. Disponível em: <http://nupet.daelt.ct.utfpr.edu.br/tcc/enge nharia/doc-equipe/2015_2_27/2015_2_27_ final.pdf>. Acesso em: 31 out. 2017.

Rüther, R. Edifícios solares fotovoltaicos: o potencial da geração solar fotovoltaica integrada a edificações urbanas e interligada à rede elétrica pública no brasil. Florianópolis: LABSOLAR, 2004. Disponível em: <http://fotovoltaica.ufsc.br/sistemas/ livros/livro-edificios-solaresfotovoltaicos.pdf>. Acesso em: 25 abr. 2017.

Seguel, J. I. L. Projeto de um sistema fotovoltaico autônomo de suprimento de energia usando técnicas MPPT e controle digital. Belo Horizonte: UFMG, 2009. Disponível em: <http://www.ppgee.ufmg.br/ defesas/216M.PDF>. Acesso em: 25 abr. 2017.

Silva, G. J; Silva, W. W. A. G.; Reis, G. L.; Rodrigues, W.A. Anais do VI Simpósio Brasileiro de Sistemas Elétricos, 2016. https://doi.org/10.20906/CPS/SBSE20160152

Simioni, T. 0 impacto da temperatura para o aproveitamento do potencial solar fotovoltaico do Brasil. Rio de Janeiro: UFRJ/COPPE, 2017. Disponível em: <http://www.ppe.ufrj.br/ppe/production/te sis/simioni.pdf>. Acesso em: 04 maio 2017.

Urbanetz Jr., J. Aspectos tecnológicos e operacionais da geração fotovoltaica. Conferência Internacional Smart Energy Paraná, 2014. 